University of Warwick institutional repository: http://go.warwick.ac.uk/wrap This paper is made available online in accordance with publisher policies. Please scroll down to view the document itself. Please refer to the repository record for this item and our policy information available from the repository home page for further information.

To see the final version of this paper please visit the publisher's website. Access to the published version may require a subscription.

Author(s): S. C. WATKINSON, L. BODDY, K. BURTON, P. R. DARRAH, D. EASTWOOD, M. D. FRICKER and M. TLALKA

Article Title: New approaches to investigating the function of mycelial networks

Year of publication: 2005

Link to published

version: http://dx.doi.org/10.1017/S0269915X05001023

Publisher statement: None 


\title{
New approaches to investigating the function of mycelial networks
}

\author{
S. C. WATKINSON ${ }^{1}$, L. BODDY ${ }^{2}$, K. BURTON ${ }^{3}$, P. R. DARRAH ${ }^{1}$, \\ D. EASTWOOD ${ }^{3}, M$. D. FRICKER ${ }^{1} \& M$. TLALKA $^{1}$
}

\author{
'Department of Plant Sciences, University of Oxford, South Parks Road, Oxford OX1 3R B \\ E-mail: sarah.watkinson@ plants.ox.ac.uk; peter.darrah@ plants.ox.ac.uk; mark.fricker@ plants.ox.ac.uk; \\ monika.tlalka@ plants.ox.ac.uk Fax: (+44 (0)1865 275074 \\ ${ }^{2}$ Cardiff School of Biosciences, Biomedical Sciences Building, M useum Avenue, PO Box 911, Cardiff CF 10 3US, UK. \\ E-mail: B oddyL@ Cardiff.ac.uk Fax: +44 (0)29 20874116 \\ ${ }^{3}$ Warwick HRI, Wellesbourne, Warwick, CV35 9EF E-mail: Kerry.Burton@ warwick.ac.uk; \\ Daniel.Eastwood@warwick.ac.uk Fax: +44 (0)2476574500
}

Fungi play a key role in ecosystem nutrient cycles by scavenging, concentrating, translocating and redistributing nitrogen. To quantify and predict fungal nitrogen redistribution, and assess the importance of the integrity of fungal networks in soil for ecosystem function, we need better understanding of the structures and processes involved. Until recently nitrogen translocation has been experimentally intractable owing to the lack of a suitable radioisotope tracer for nitrogen, and the impossibility of observing nitrogen translocation in real time under realistic conditions. We have developed an imaging method for recording the magnitude and direction of amino acid flow through the whole mycelial network as it captures, assimilates and channels its carbon and nitrogen resources, while growing in realistically heterogeneous soil microcosms. Computer analysis and modeling, based on these digitized video records, can reveal patterns in transport that suggest experimentally testable hypotheses. Experimental approaches that we are developing include genomics and stable isotope NMR to investigate where in the system nitrogen compounds are being acquired and stored, and where they are mobilized for transport or broken down. The results are elucidating the interplay between environment, metabolism, and the development and function of transport networks as mycelium forages in soil. The highly adapted and selected foraging networks of fungi may illuminate fundamental principles applicable to other supply networks.

Keywords: Fungal development, fungal translocation, nitrogen cycling

Mycelial networks sustain forest ecosystems, which occupy a third of the Earth's land surface. Fungal mycelium dominates the microbial biomass of forest soils, commonly contributing $70-110 \mathrm{~g}$ dry wt $\mathrm{m}^{-2}$ (Markkola et al., 1995). Fungi are the major agents of decomposition and play a key role in nutrient cycles as saprotrophs and mycorrhizal symbionts. Central to their importance is the extent that they influence control of the nitrogen and phosphorus supply (Boddy \& Watkinson, 1995; Dighton, 1997). Microbial biomass can accumulate and retain mineral nutrients, immobilising as much as $20 \%$ and $18 \%$ respectively of the total soil nitrogen and phosphorus (Bääth \& Söderstrom, 1979). Mycelia scavenge and sequester nutrients from soil, concentrate mineral nutrients from plant litter that they decompose (Sen, 2000), relocate nutrients between different organic resources, and ultimately make nutrients available to plants via the 'wood-wide web' to maintain primary productivity (Read, 1997). It is believed that the spatial integrity of the networks may be critical for ecosystem function, by preventing nutrient loss by leaching (Miller \& Lodge, 1997; Swift \& Anderson, 1996).

There is evidence that fungi are capable of affecting the rate of release of sequestered carbon by collecting and transporting nitrogen/phosphorus to freshly colonized woody resources (Boddy \& Watkinson, 1995). Lignocellulosic plant remains are carbon-rich but nitrogen-poor, with decomposition rates in the field correlating with N content (Dighton, 1997). Mycelial import of nitrogen accelerates the decomposition of pure cellulose in microcosms designed to mimic cellulosic plant litter being colonized by mycelium 


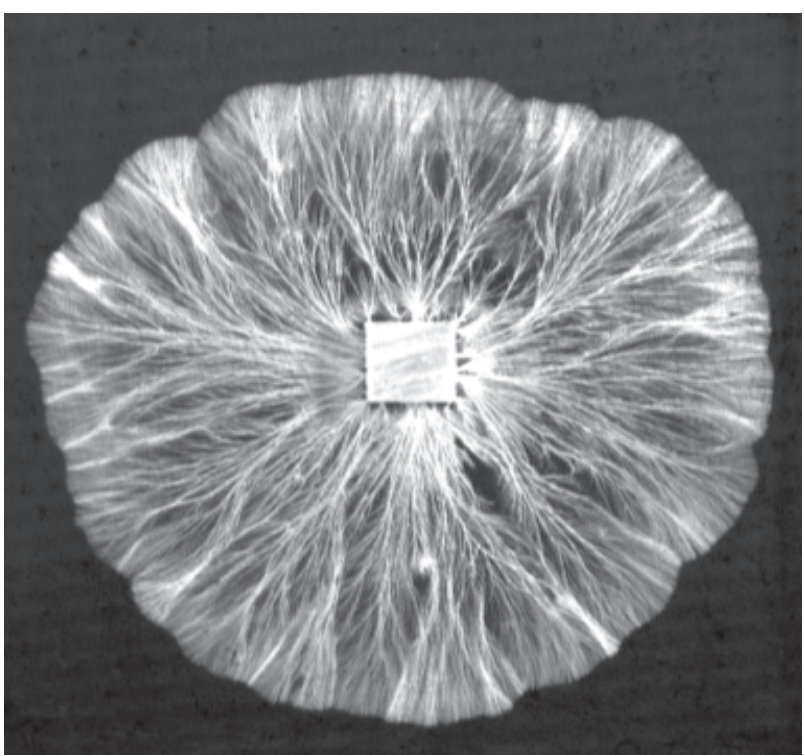

Fig 1 Digital image of a mycelial system of Hypholoma fascicularegrowing from wood block of $2 \mathrm{~cm}$ side in $24 \times 24 \mathrm{~cm}$ tray of compressed non-sterile soil. Mass fractal dimension $>1.9$. Photo by George Tordoff.

growing into it from underlying $\mathrm{N}$-rich soil (Watkinson et al., 1981). Fungicide treatment of field soil reduces the nitrogen content of surface plant litter compared with untreated controls, and reduces decomposition rate (Frey et al., 2000). However, quantifying fungal nitrogen translocation in mycelium, and predicting its contribution to decomposition in ecosystems, presents formidable methodological problems.

There is good understanding of the physiology underlying nitrogen acquisition and metabolism and its regulation from 'laboratory' species such as yeast, Neurospora and Aspergillus (Griffin, 1994). Fungi actively scavenge nitrogen and are equipped with a remarkable complement of constitutive and induced membrane transport systems in the plasmalemma and tonoplast (Klionsky et al., 1990), enabling them to acquire and store both inorganic and organic nitrogen opportunistically wherever they encounter it in the course of their growth. It is accumulated first in expandable pools of free, soluble amino acids (Griffin, 1994). Close control on biochemical pathways of nitrogen metabolism is operated through transcription regulating genes, for example NIT2 of Neurospora and AREA of Aspergillus nidulans, that regulate the expression of genes for enzymes of nitrogen assimilation and catabolism according to whether the mycelium is experiencing nitrogen starvation or glut (Griffin, 1994; Marzluf, 1996).

The main difference between growth of model fungi such as Aspergillus and Neurospora in routine laboratory culture, and fungi living in real ecosystems, is in the

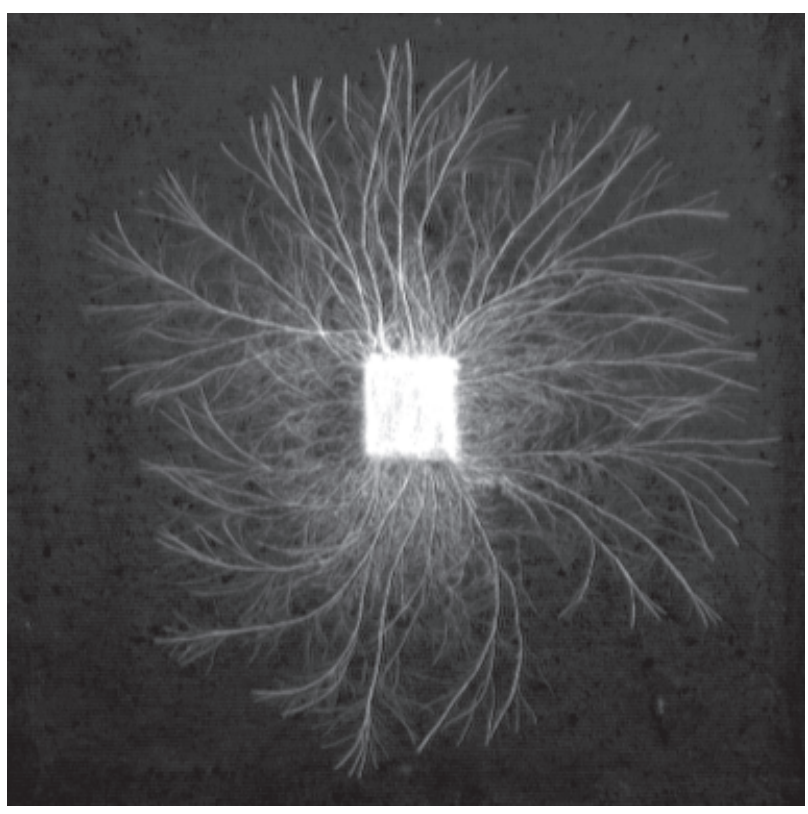

Fig 2 Mycelial system of Phanerochaetevelutina in soil as in Fig 1. Mass fractal dimension approx. 1.6. Photo by George Tordoff.

structural complexity and duration of mycelial networks. In large basidiomycete colonies growing in natural habitats, both starvation and excess are likely to be encountered at the same time in different parts of the network. Basidiomycetes typically are able to translocate water and solutes long distances with in the hyphae (Jennings, 1987), allowing exploratory outward growth from a food base over a nutritionally inert surface. Many cord-forming species develop persistent networks (Thompson, 1984) with specialised high-conductivity channels - mycelial cords - that carry nutrients between separate carbon-rich food resources. Wood decay basidiomycetes concentrate nitrogen acquired from wood and other sources, accumulating it opportunistically in excess of requirements in the form of free amino acids stored in cytoplasm and vacuoles (Venables \& Watkinson, 1989). These amino acids are transported in cords, and become incorporated into protein and cell walls. Development in such fungi is closely linked to the $\mathrm{C} / \mathrm{N}$ ratio and the intracellular nitrogen concentration within the mycelium (Watkinson, 1999).

New techniques are needed that can probe the full range of developmental, physiological and biochemical responses during the establishment and maintenance of complex networks under realistic conditions. Fungi have to grow to feed, and the adaptive development of the mycelium as it encounters and exploits its scattered woody food sources is described as 'foraging'. Modern imaging methods can now be combined with mathematical modelling to investigate development of 


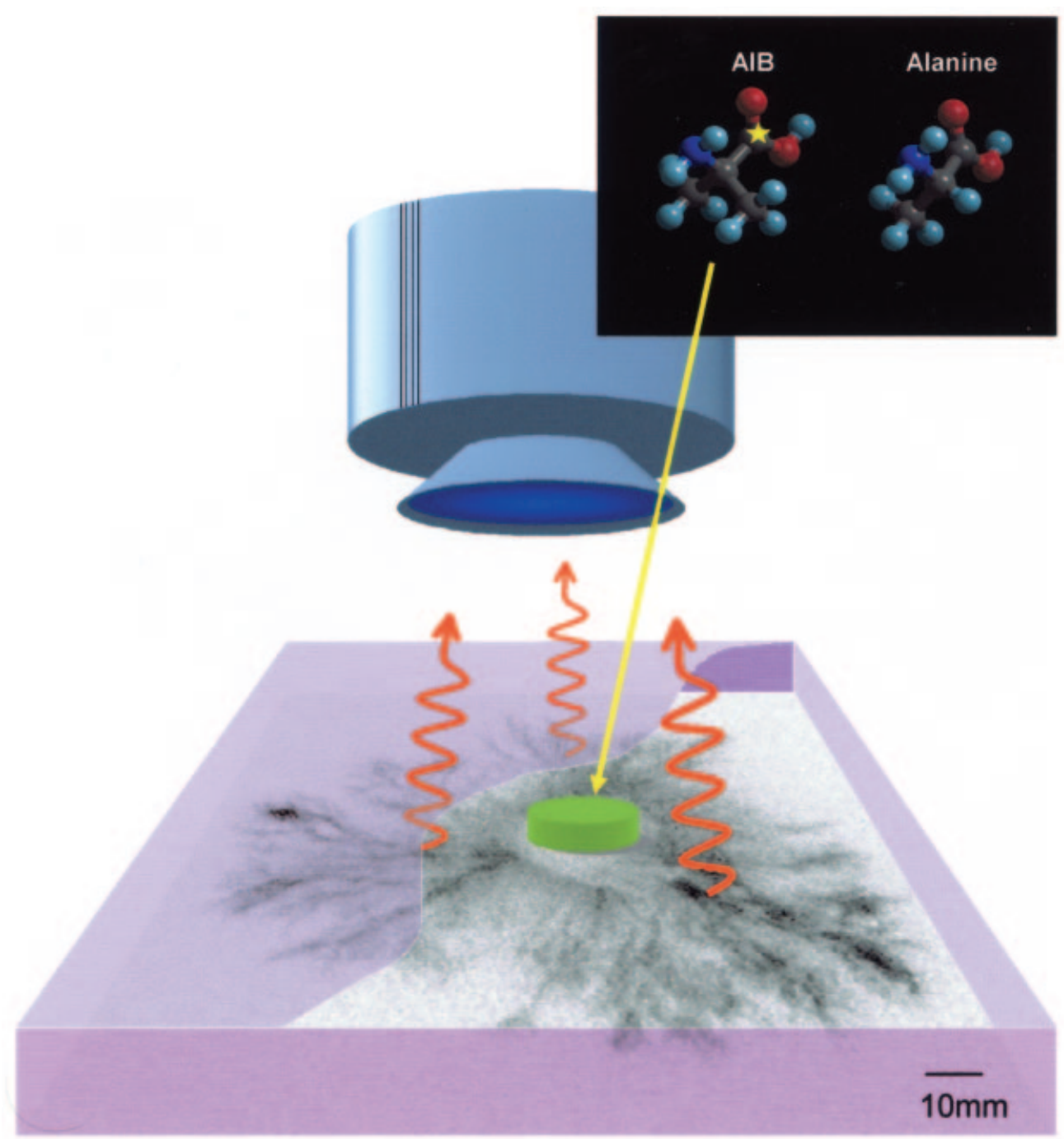

Fig 3 Photon counting camera system used to image transport of ${ }^{14} \mathrm{C}$-labelled (yellow star) non-metabolized amino acid analogue, AIB, through the mycelium of Phanerochaete velutina growing over an intensifying screen in a microcosm.

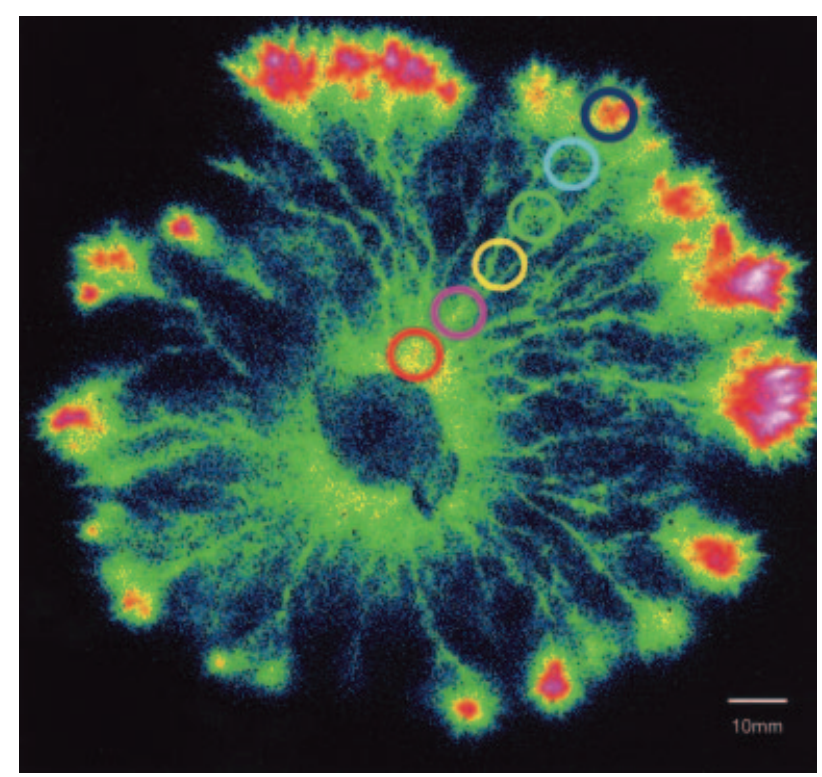

Fig 4 Distribution of ${ }^{14} \mathrm{C}$-labelled AIB through the mycelium of $P$. velutina.

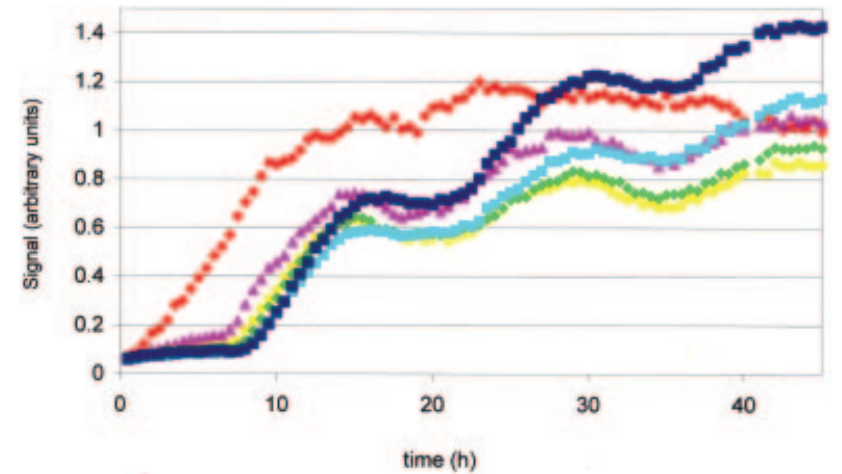

Fig 5 Regular pulses in transport recorded at the sites indicated in Fig 4.

foraging mycelium, and explore the path of nutrients into hyphae and throughout the network. Based on imaging techniques, theoretical models can generate precise hypotheses for experimentation. With current methods in molecular gen etics (e.g. Breeze et al., 2004), 
it is now possible experimentally to compare the expression levels of genes with known metabolic functions, offering a new way of tracking changes in the metabolic state of the organism. Further, mathematical description of development in mycelium is advancing our understanding of the evolutionary adaptations in fungal development for foraging in the patchy, unpredictable nutrient supply of real ecosystems.

Mycelial cords, the main transport pathways in mycelial networks of those woodland fungi not confined to a single resource unit (e.g. a stump or twig), develop in a way that suggests each cord-forming species has evolved a different foraging strategy. Long range foragers such as Phanerochaete velutina grow fast for long distances, with cords developing early in growth to form long supply lines from the large wood food bases this organism prefers. Advance only halts when the mycelium meets, and pauses to exploit, a substantial new wood resource (Boddy, 1999). By contrast, Hypholoma fasciculare, which lives on dead leaves as well as substantial pieces of wood, forages over a short range, spreading mainly as fanned-out mycelium composed of separate hyphae, with shorter cords. The use of fractal dimension (Boddy et al., 1998) to describe the organisation of the mycelial network, and its responses to various baits as it grows in suitable microcosms, can highlight developmental shifts - some so subtle as to evade simple observation - between diffuse assimilative and corded distributive growth. The fractal dimension provides a means to convey these patterns in quantitative form and to measure changes (Figs 1 and 2). Essentially, it measures space-filling, and allows description of structures that do not take Euclidean whole number dimensions of 1 (straight lines), 2 (flat surfaces) or 3 (volumes), but rather have fractional dimensions between these.

Cords perform a remarkable range of transport functions, carrying carbon sources including sugars and polyols, amino acids, phosphate and water (Cairney, 1992; Boddy, 1999; Jennings, 1987; Watkinson, 1984). Substances can travel towards the growing margin and back to base at the same time, although not necessarily by the same transport system. However, we know little about the cellular and subcellular anatomy of the pathway, the mechanism of transport and its driving forces, and the information pathways through mycelium implicit in the coordinated system-wide responses that it displays in response to localised nutritional stimuli. One approach to measuring transport of solutes is to follow the movement of radio-labelled compounds through the mycelium. Often the final radiolabel distribution is visualized using autoradiography techniques or analysed by destructive harvesting of the tissues followed by scintillation counting. To try to provide a more dynamic view of how the transport system develops and alters, we are exploring the use of a new technique, termed Photon Counting Scintillation Imaging (PCSI), to image amino acid translocation continuously in foraging mycelium (Tlalka et al., 2002; Fig 3). Amino acid is chosen because nitrogen translocation by mycelium is a pivotal ecosystem process, and amino acid is presumed to be the chemical form in which accumulated nitrogen is translocated through hyphae, since it is soluble, mobile in mycelium, and is the form in which nitrogen acquired by the mycelium is stored until being converted into protein and used for growth. The existence of an amino acid, 2aminoisobutyric acid (AIB), which is actively accumulated in the fungus, but neither lost as $\mathrm{CO}_{2}$ by metabolism nor incorporated into protein (Watkinson, 1984), allows amino acid translocation to be tracked through the mycelial system using a ${ }^{14} \mathrm{C}$-label as marker - nitrogen itself has no convenient radioisotopes, so cannot be tracked directly.

In the PCSI technique, the fungus is grown in close proximity to a scintillation screen and the movement of the radiolabelled compound tracked from the photons of light that result from collision of the radioactive emissions from the compound with the screen. Using an extremely sensitive photon-counting camera it is possible to collect time-lapse images at intervals of 30 $60 \mathrm{~min}$ for periods in excess of 4 weeks. In simple microcosms with mycelium growing out over a screen from a central inoculum, transport occurred towards the margin of the colony, concentrated in cords if these were present (Fig 4). The signal from different regions of the mycelium can be quantified from these images (Fig 5) and revealed that there was a strong pulsatile component associated with rapid transport, particularly through the corded system. Even faster rates of transport and more pronounced oscillations were observed in larger colonies grown across natural substrates such as sand and soil.

Many biological systems show pronounced oscillations with a period of around 24 hours and are termed circadian (circa, diem). These are often associated with anticipatory behaviour to adjust the metabolism and physiology of the organism to predictable changes in the environment such as daybreak. Such circadian rhythms are driven by a central 'clock' and are not affected by changes in temperature. In contrast, the oscillations observed here are significantly frequent than $24 \mathrm{~h}$ and alter markedly with temperature, the frequency doubling about every 
$10^{\circ} \mathrm{C}$. This suggests that they arise directly from a metabolic process rather than reflect the underlying rhythm of the 'clock'. The frequency of the pulses was established using Fourier techniques. Fourier analysis is a family of mathematical techniques, all based on decomposing signals into sinusoids, each with a particular frequency, magnitude and phase. For a fairly regular oscillation, the dominant Fourier frequency provides a very convenient and compact description of the signal that captures most of its inter esting features. On more detailed examination, the shape of the pulses was highly asymmetric, with an apparent switch between a gradual rise and a sudden drop in the intensity of the amino acid signal during each cycle. Furthermore, exactly the opposite profile was observed from the assimilatory hyphaegrowing on the inoculum compared to those that were foraging (Tlalka et al., 2003; Fig 6). This suggested one plausible sequence of events to explain these data that is consistent with evidence for amino acid transport systems in the literature. Signal would initially increase with uptake of amino acid into cytoplasm and sequestration into vacuoles. Once these have reached a threshold level, a proportion of the amino acid would be released into a pathway for mass flow to the growing hyphae at the colony margin. A computer simulation model was constructed incorporating partial differential equations describing the concentration dependence of these processes, which generated a comparable pulse pattern to that observed when values based on physiological data were used for amino acid transport rates across plasmalemma and tonoplast (Tlalka et al., 2003). Whether such a model is sufficient to explain all the experimental data remains to be established, however, at this stage it is consistent with the vacuole as a key compartment in amino-acid dynamics in mycelial fungi.

A role for the vacuole in solute transport has already been suggested for a number of mycorrhizal fungi by A shford and colleagues (A shford et al., 2001) after they observed a highly dynamic tubular network using fluorescence microscopy. A similar dynamic network of tubular vacuoles interconnecting systems of larger and constantly changing vacuoles was found in Phanerochaete velutina using confocal scanning laser microscopy (CSLM; Fig 7). Simultaneous use of dyes preferentially accumulated in other subcellular compartments, such as mitochondria (Fig 8), is helping us to understand whether this dynamic behaviour is specific to vacuoles or a general feature of an extremely active cytoplasm. An important technical challenge is to apply similar microscopy techniques to study the internal structure of hyphae which have ceased to grow, but are in the process of differentiating to form the very rapid, high conductivity pathway through mycelial cords revealed in PCSI.

Whilst PCSI and CSLM are proving useful tools to visualize dynamic processes, they can provide relatively little information on the biochemical processes that are normally associated with $\mathrm{N}$-uptake and metabolism and how these processes may alter with changing nutrient supply and demand. We intend to apply stableisotope NMR to profile the underlying metabolic pathways, and to test the hypothesis (Bago et al., 2001) that the urea cycle is invoved in vacuolar nitrogen transport. These studies will be linked with characterization of the molecular control systems for nitrogen distribution during mycelial foraging, using methods for fungal gene expression of nitrogen metabolism and mobilisation developed at WarwickHRI (Eastwood et al., 2001). Thus our experiments in 'spatial metabolomics' are designed to map the transcript level of relevant genes on to the patterns of mycelial differentiation and amino acid transport imaged during foraging development of mycelium in baited microcosms. The metabolic differentiation of assimilative and exploratory mycelium predicted by models based on imaging, will be tested by comparing levels of expression of genes coding for enzymes involved in nitrogen acquisition, metabolism and mobilisation.

While we expect that this multi-disciplinary approach will facilitate a much more comprehensive description of nitrogen cycling in ecologically important basidiomycetes, it also represents an opportunity to dissect how a self-organising, complex biological network develops. At a more abstract level, such a network represents a generic class of complex systems with loose parallels across a broad range of physical, biological and socio-economic fields. Complex systems in this context can be characterized as consisting of interacting populations (or networks) of autonomous and adaptive agents (or components) which allocate global resources with reasonable efficiency on the basis of incomplete and noisy information without the need for a central control mechanism. Examples of such systems permeate the physical, biological and social sciences, and include traffic flow, internet routing, the response of the immune system, financial markets and manufacturing supply chains. It is possible that the study of nutrient fluxes in such superbly well-adapted mycelial networks can help us understand how efficient resource allocation and robustness can be achieved in manmade networks, despite non-linear dynamics and the fact that the transmitted information is limited and noisy. 

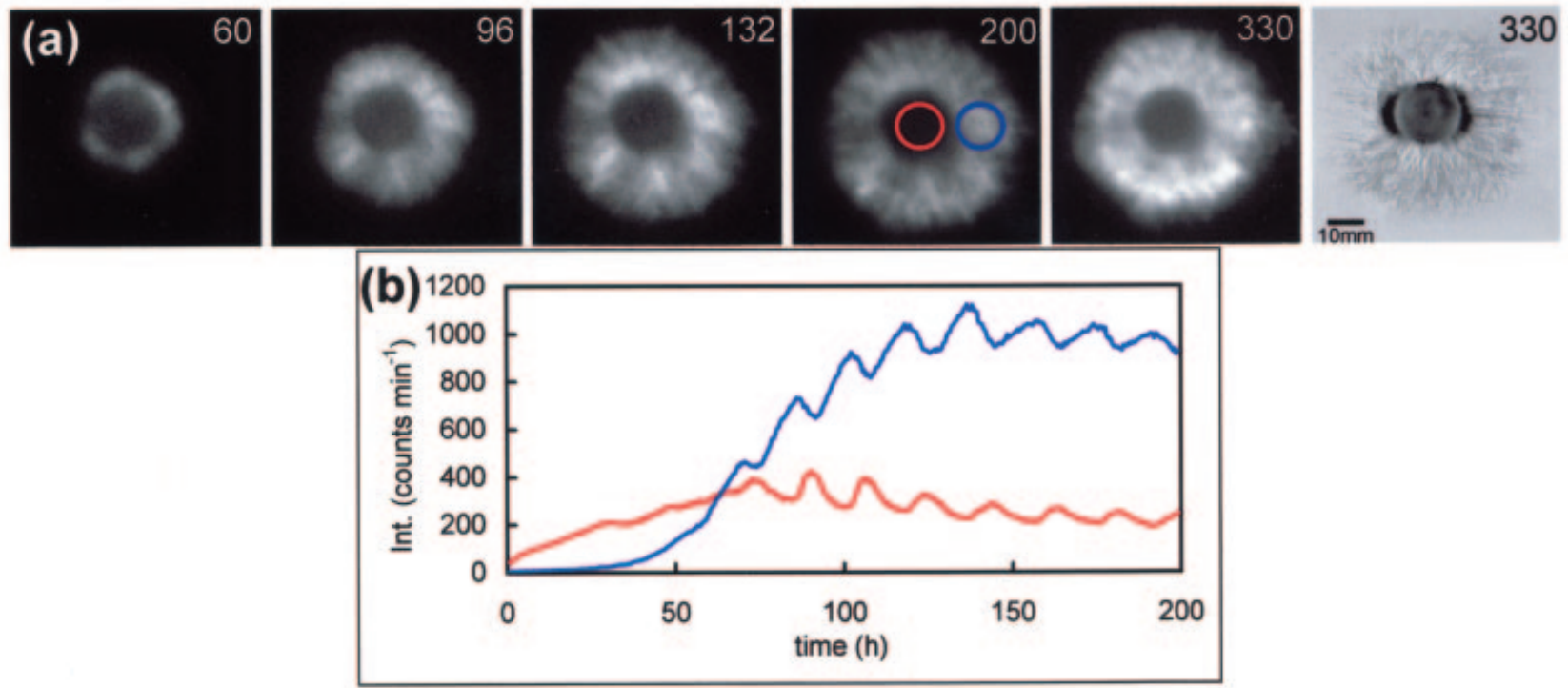

Fig 6 a Photon counting scintillation images of mycelium of P. velutina growing over glass from a central inoculum, with brightfield image shown at $330 \mathrm{~h}$. Fig 6 b Pulse profiles from inoculum and outgrowing mycelium, at sites indicated on image at 200 h in Fig 6 a.

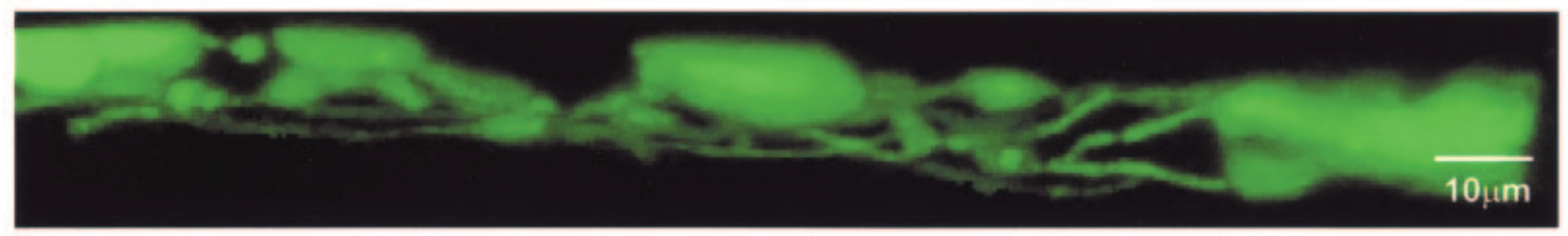

Fig 7 Dynamic vacuolar network - a possible nutrient transport channel - stained with Oregon Green

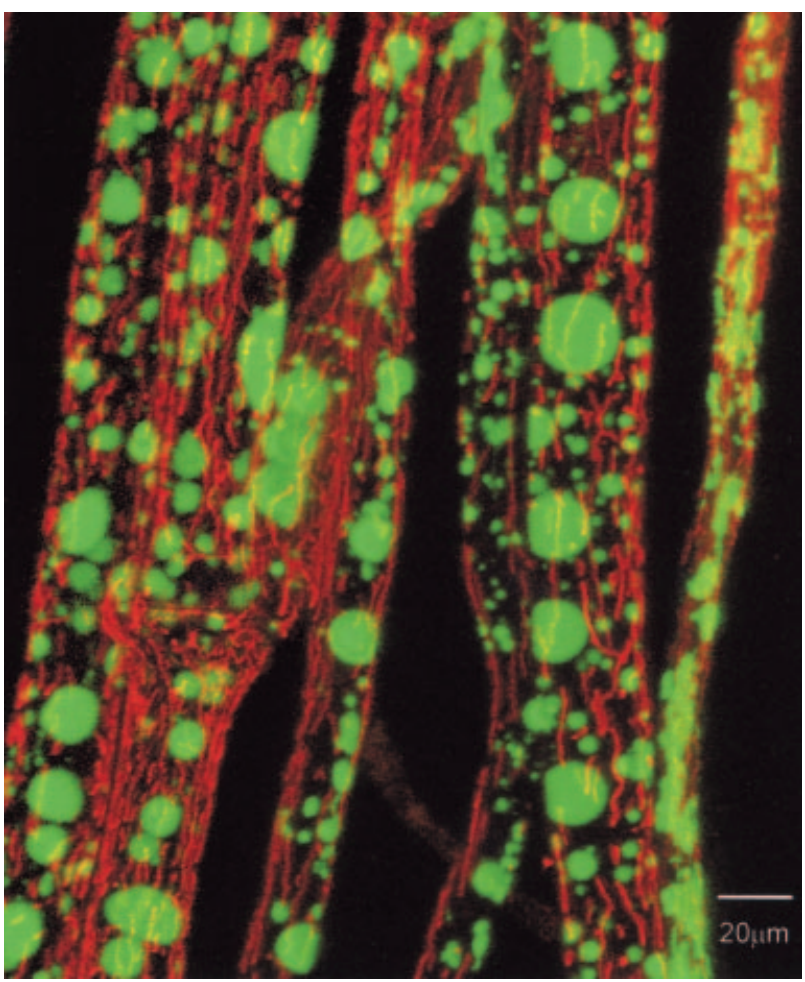

Fig 8 3-D confocal image of P. velutina vacuoles (green) and mitochondria (red) labelled with fluorescent dyes Oregon Green and Rhodamine B.

\section{Acknowledgements}

The authors are grateful for funding from the Natural Environment Research Council, the Biotechnology and Biological Sciences Research Council, the Royal Society, the British Mycological Society and the Dunston bequest of the University of Oxford.

\section{References}

Ashford, A.E., Cole, L. \& Hyde, G.J. (2001). Motile tubular vacuole systems. In The M ycota VIII: Biology of the Fungal Cell, eds. R.J. Howard \& N.A.R. Gow Springer-Verlag, Berlin. Bääth, E. \& Söderström, B (1979). Fungal biomass and fungal immobilisation of of plant nutrients in Swedish coniferous forest soils. Revue d'Écologie et de Biologie du Sol 16: 477-489.

Bago, B., Pfeffer, P. \& Shachar-Hill, Y (2001). Could the urea cycle be translocating nitrogen in the arbuscular mycorrhizal symbiosis? New Phytologist 149: 4-8.

Boddy, L. (1999) Saprotrophic cord-forming fungi: meeting the challenge of heterogeneous environments. M ycologia 91: 13 - 32

Boddy, L. \& Watkinson, S.C. (1995). Wood decomposition, higher fungi and their role in nutrient redistribution. Canadian Journal of B otany 73: S1377-S1383.

Boddy, L., Wells, J.M., Culshaw, C. \& Donnelly, D.P. (1998). Fractal analysis in studies of mycelium in soil. Geoderma 88: 301-328. 
Breeze, E., Wagstaff,C., Harrison, E., Bramke, I., Rogers, H., Stead, A., Thomas, B. \& Buchanan-Wollaston, V. (2004). Gene expression patterns to define stages of post-harvest senescence in Alstroemeria petals. Plant Biotechnology Journal 2, 155-168.

Cairney, J.W.G. (1992) Translocation of solutes in ectomycorrhizal and saprotrophic rhizomorphs. M ycological Research 96: 135-141.

Dighton, J. (1997). Nutrient cycling by saprotrophic fungi in terrestrial habitats. In: Environmental and M icrobial Relationships eds. D.T. Wicklow \& B.E. Soderstrom. The M ycota VI pp.271-279.

Eastwood, D. C., Kingsnorth, C. S., Jones, H. E. \& Burton, K. S. (2001). Genes with increased transcript levels following harvest of the sporophore of Agaricus bisporus have multiple physiological roles. M ycological Research 105: 1223-1230.

Frey, E. T. Elliott, K. Paustian \& G. A. Peterson (2000) Fungal translocation as a mechanism for soil nitrogen inputs to surface residue decomposition in a no-tillage agroecosystem, Soil Biology and Biochemistry, 32: 689-698

Griffin, D.H. (1994). Fungal Physiology, $2^{\text {nd }}$ edn. Wiley-Liss, New York.

Jennings, D.H. (1987). The translocation of solutes in fungi. Biological Reviews 62: 215-143.

Klionsky, D.T., Herman, P.K. \& Ek, H. (1990). The fungal vacuole: composition and biogenesis. Microbioogical Reviews 54: 226-292.

Markkola, A. M., Ohtonen, R., Tarvaien, O. \& A honenJonnnarth, U. (1995). Estimates of fungal biomass in Scots Pine stands on an urban pollution gradient. New Phytologist 131: 139-147.

Marzluf, G.A. (1996). Regulation of nitrogen metabolism in mycelial fungi. In: R. Brambl \& G. A. Marzluf, eds. B iochemistry and M olecular B iology. The M ycota eds. Esser K. \& Lemke P.A . III: 357-368.

Miller, R. M. \& Lodge, D. J. (1997). Fungal responses to disturbance: Agriculture and Forestry. In: D. T Wicklow \& B. Soderstrom, eds. Environmental and M icrobial Relationships. The Mycota VI, 65-84.
Read, D. (1997). The ties that bind. Nature 388, 517-518.

Sen, R. (2000). Budgeting for the wood-wide web. New Phytologist 145: 161-163.

Swift, M. \& Anderson, J. (1996). Biodiversity and ecosystem function in agricultural systems. In E.D. Schulze, \& H.A. Mooney, eds: B iodiversity and E cosystem Function. Springer.

Thompson, W. (1984). Distribution, development and functioning of decomposer basidiomycetes of the deciduous woodland floor. In D.H. Jennings \& A.D.M. Rayner eds. The Ecology and Physiology of the Fungal M ycelium. Cambridge University Press.

Tlalka, M., Watkinson, S. C., Darrah, P. R. \& Fricker, M. D. (2002) Continuous imaging of amino acid translocation in intact mycelia of Phanerochaete velutina reveals rapid, pulsatile fluxes. New Phytologist 153: 173-184.

Tlalka, M., Hensman, D., Darrah, P. R., Watkinson, S. C. \& Fricker, M. D. (2003). Noncircadian oscillations in amino acid transport have complementary profiles in assimilatory and foraging hyphae of Phanerochaete velutina. New Phytologist 158: 325-335.

Venables, C. E. \& Watkinson, S. C. (1989). Medium-induced changes in patterns of free and combined amino acids in mycelium of Serpula lacrymans. Journal of General M icrobiology 135: 1369-1374.

Watkinson, S.C. (1984). The inhibition of growth and development of Serpula lacrymans by the non-metabolised amino acid analogue 2-aminoisobutyric acid. FEMS M icrobiology Letters 24: 247-250.

Watkinson, S. C. (1999). Metabolism and hyphal differentiation in large basidiomycete colonies. In The Fungal Colony, eds N. A . R. Gow \& G. M. Gadd, Cambridge University Press, Cambridge.

Watkinson, S. C., Davison, E. M. \& Bramah, J. (1981). The effect of nitrogen availability on growth and cellulolysis by Serpula lacrymans. N ew Phytologist 89: 295-305. 